\title{
Research on SVPWM Method and its Neutral Point Potential Control
}

\author{
Yan Liu ${ }^{1, a}$, Xu Wang ${ }^{2, b}$, Yan Xing ${ }^{3, c}$ \\ Detection Technique and Automatic Device, College Of Information Science And Engineering \\ Northeastern University, Shenyang Liaoning Province \\ Email: Iyfx0418@yahoo.cn
}

Keywords: Neutral-point-clamped inverter, SVPWM (space vector pulse width modulation), Neutral Point Balance

\begin{abstract}
Neutral-point potential unbalance is an inherent problem of Neutral-point-clamped three-level PWM rectifiers. If the problem of neutral point can't be controlled appropriately, the harm of input current will increase, and even the dc-link capacitor and switches will be destroyed. Thus domestic and foreign experts have done lots of research on the balance control of Neutral-point-clamed and put forward many effective control methods.

This paper proposes a novel SVPWM strategy for the three-level neutral-point-clamped voltage source inverter, based on the particular disposition of all the redundant voltage vectors. The new modulation approach shows superior performance for the harmonic voltage and the control of neutral-point potential compared to the popular eight-stage centered SVPWM and realizes the balancing control of inverter neutral-point potential by modifying redundant small vectors pairs' distribution factor accurately, only requiring the information of dc-link capacitor voltages and three-phase load currents, which is convenient to apply and is compatible of digital computer realization. The feasibility of the proposed control approach has been verified via simulation and experiment results.

In the strategy of software control, domestic and foreign scholars propose numerous neutral point potential control schemes. When carrier modulation is used, the balance control is achieved by injecting zero-sequence component into the three-phase modulated wave. When space vector modulation methods are adopted, they can be divided into three categories: passive control, hysteresis control and active control. there is also a new algorithm based on the intrinsic relationship between SVPWMs for two-level inverters and three. 1evel inverters. a novel SVPWM control algorithm is proposed for three. 1 evel. The dwell time of voltage vector for three-level inverter can be acquired from counterpart for two-level inverter by using a linear transformation. Aiming to analysis the output PWM sequence of three-level inverter, a novel classification standard of voltage vector is proposed. Based on evaluating the PWM sequences, a novel PWM sequence is given, that can reduce the total harmonics distortion of inverter output.
\end{abstract}

\section{Introduction}

Three-level inverters are widely used in industrial high-voltage adjustable speed drives. The three-level inverters are very rapidly growing area of power electronics which will be good potential in the future. Compare to two-level inverter it owns the advantage as follows:

$\checkmark$ Compare to two level inverter, three-level inverter can reduce the stress on switching devices.

$\checkmark$ The three level circuit can reduce harmonic wave .The output wave is nearly to be sinusoidal wave.

The three-level inverter can operation the switch device on the low frequency. 
The main circuit of three level is shown in Fig.1. As shown in Fig. 1, the inverter produces the three electrical potentials by the use of the two DC-link capacitances in series, and so long as the characteristic of the two capacitors themselves are not identical or three-phase electric current is not symmetrical.Because of this, he three-level topology has an inherent problem, namely, the imbalance of neutral-point potential. If the voltages on the DC-link capacitors cannot get balance, it will make the output voltage contain second or higher order even harmonic. This will result in serious damage to power devices, thus limiting its application.

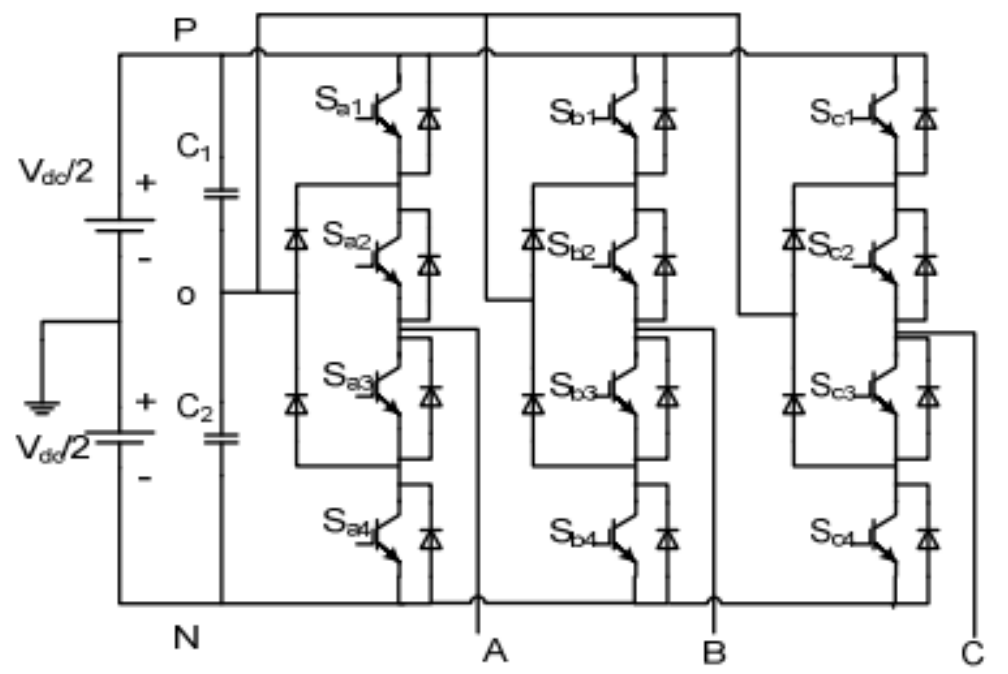

Fig. 1. The Three-level Inverter Main Circuit

This paper analyzes the main reason of imbalance of capacitor voltage and proposes a neutral-point potential control method by factor. This method has simple computation and strong robust features. Simulation and experimental results show that this method can effectively control the three-level inverter capacitor voltage imbalance.

\section{The Supwm Method}

There are two methods The space vector diagram is shown in Fig. 2 of a three-level inverter is made up of 19 switching vectors and 27 switching states, which can be divided into six regions from A to $\mathrm{F}$ and each region also can be partition into four triangles. The basic vectors are separated into four kinds of basic vectors for 27 kinds of switching states.

The switching vectors can be divided into four types of vectors; zero vector, short vectors, medium vectors and large vectors. The zero vector and large vector do not affect the neutral point balance. However, short and medium vectors affect the neutral point balance. Any short vector has two switching states. One state increases Uc2, while the other decreases it. Therefore, by adjusting the duty ratios of these two states, the neutral point fluctuation can be minimized. A medium vector has only one switching state. Therefore, unlike short vectors, medium vector does not have balancing capability.

There are two kinds of small vectors, the influence of which to the neutral-point potential is opposite, but they are for the same vector in the space vector pattern as showed in Fig. 2. So we can choose different switching states to compose a small vector. That is why we could regulate the balance of the neutral-point potential by means of choosing the dwelling time of different small vectors. From the analysis above we know that the fluctuating of the neutral-point potential is due to the neutral-point current. It is the charge and discharge of the two DC-link capacitors that cause the fluctuating. Suppose that the capacitors are fixed, the fluctuating range of the neutral-point potential 
lies on the ampere, direction and dwelling time of the neutral-point current, which have relation with the three switching states of the three phases. Table showed the switching states of middle vectors and small vectors with their relevant neutral-point current.

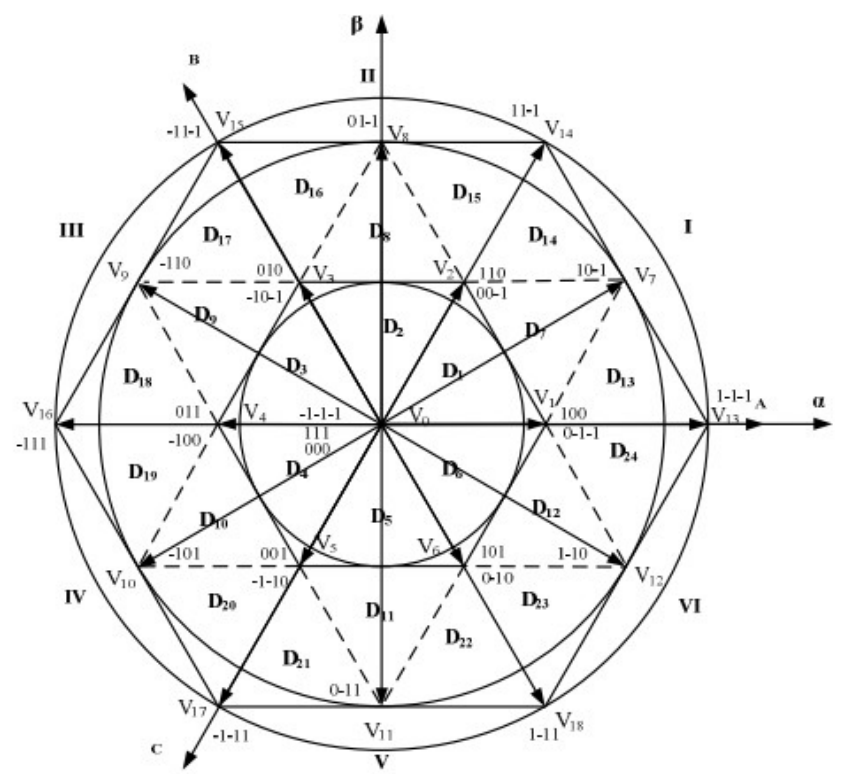

Fig. 2. Three-Level Space Vector Diagram

Table1 A Three-Level Inverter Switching States

\begin{tabular}{cccccc}
\hline Switching states & S1 & S2 & S3 & S4 & SN \\
\hline P & ON & ON & OFF & OFF & Vd \\
O & OFF & ON & ON & OFF & Vd/2 \\
N & OFF & OFF & ON & ON & 0 \\
\hline
\end{tabular}

\section{Simulation}

Now we simulation the three level inverter system with the software matlab.the DC voltage is 600 V.Frequence is 50hz. Fig3 shows the whole system.

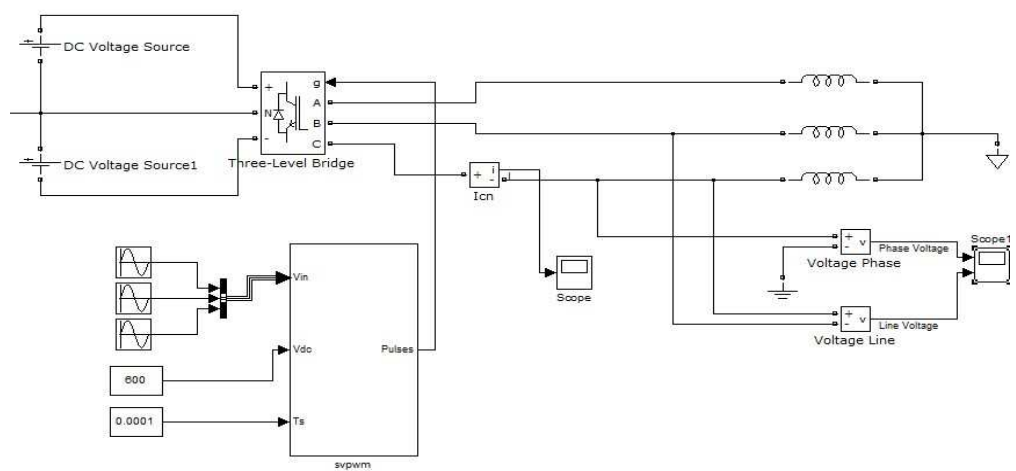

Fig. 3. The Three-level inverter System

The proposed method is simulated for strategy with and without neutral point potential balance control to verify the effectiveness. We adopt the Svpwm to produce the wave of PWM. Fig. 5 and Fig. 6 show simulation results of phase voltage and line voltage 


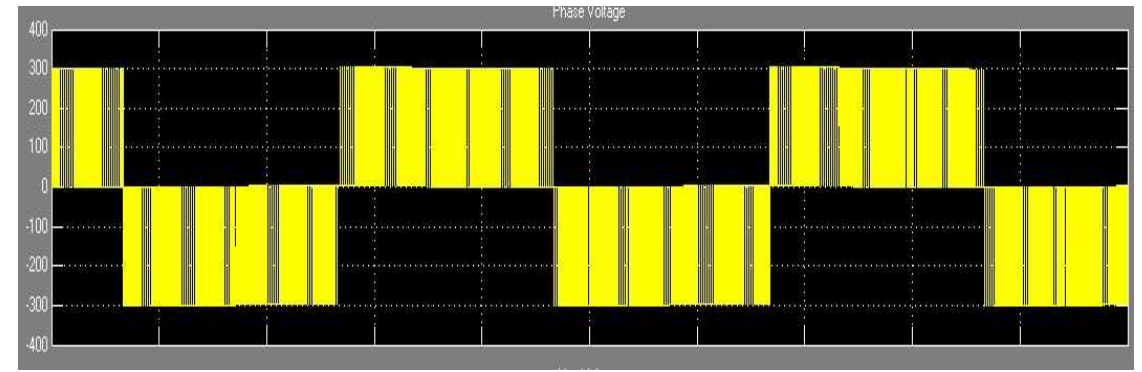

Fig. 4. The phase voltage

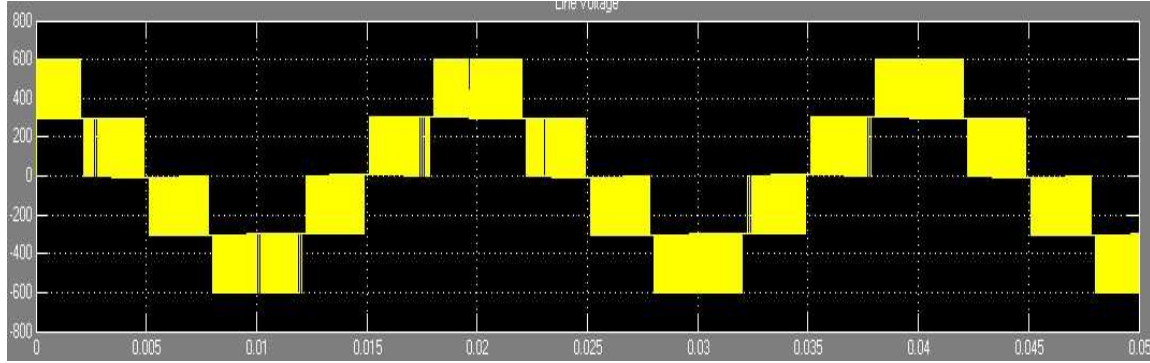

Fig. 5. The line voltage

\section{Conclusion}

In this paper, we research on the three-level inverter system and the unbalanced causes of the neutral point potential and the influence of middle and small vectors. The algorithm we adopt has better robustness and adaptability for time-variable nonlinear systems. The result shows the effective of the algorithm.

\section{References}

[1] Kai Yan, Wei Wang, Study On Neutral-point Balancing For Three-level Space Voltage Vector Pulse-width modulation Inverter. Electrical Machines and Systems, 2008.ICEMS 2008. International Conference . 1571-1576.

[2] R Krishna, E S Deepak, Sasi K Kottayil, Mats Leijon, Space Vector Modulation Method to Balance theNeutral Voltage for a Three Level Inverter. Power Electronics, Drives and Energy Systems (PEDES) \& 2010 Power India, 2010 Joint International Conference.1-6 